\title{
Integrating topological degrees of freedom with perturbation theory in three dimensional Yang-Mills theory
}

\section{Chandrasekhar Chatterjee*}

The Institute of Mathematical Sciences, C.I.T. Campus, Taramani P.O., Chennai 600113, India

E-mail: chandra@imsc.res.in

\section{Indrajit Mitra}

Department of Physics, University of Calcutta,

92 A.P.C. Road, Kolkata 700009, India

E-mail: indrajit.mitra@saha.ac.in, imphysecaluniv.ac.in

\section{H. S. Sharatchandra}

The Institute of Mathematical Sciences, C.I.T. Campus, Taramani P.O.,

Chennai 600113, India

E-mail: sharateimsc.res.in

\begin{abstract}
Polyakov has demonstrated that a monopole plasma gives confinement in Georgi Glashow model in three Euclidean dimensions. However his semi classical techniques are not applicable for pure $\mathrm{YM}_{3}$. Even though the gauge potential ansatz of 't Hooft Polyakov monopole has a finite action in $\mathrm{YM}_{3}$, it is not a stable classical solution, indeed, larger the 'size' smaller the action.

We develop techniques for summing over such large overlapping monopole configurations. This is based on i) characterizing the configurations using eigenfunctions of $B_{i}(x) \cdot B_{j}(x)$, ii) obtaining a two potential formalism for Yang Mills theory. These techniques provide a way for combining renormalized perturbation theory and asymptotic freedom with confining effects of fluctuations of topological significance.
\end{abstract}

Sixth International Conference on Quarks and Nuclear Physics,

April 16-20, 2012

Ecole Polytechnique, Palaiseau, Paris

\footnotetext{
${ }^{*}$ Speaker.
} 


\section{Introduction}

Quantum Chromodynamics is accepted as the theory of strong interactions. Renormalizability and asymptotic freedom make the theory well under control. It is supposed to confine quarks in the infrared. Strong coupling expansion of lattice gauge theory and computer simulations are supporting this fact. However, the confinement mechanism is not understood fully. There are very few firm insights into this aspect. Topological configurations are believed to play a crucial role but calculational schemes are not available in the literature. However, in 2+1 dimensional GeorgiGlashow model confinement has been shown by Alexander Polyakov [1] using non perturbative calculation. The perturbative spectrum of Georgi Glashow Model consists of a massless photon, massive charged vector bosons and a massive Higgs. However, non-perturbative effects change the spectrum of the theory drastically. The action has a stable, topologically non-trivial extremum and it is called the 't Hooft-Polyakov monopole (playing the role of an instanton here). In a certain regime of coupling constant the partition function can be written as a partition function of grand canonical ensemble of dilute monopole gas. In this regime of coupling constants, a dilute gas approximation of (anti)monopole plasma can be justified and the effects can be reliably computed. The 'plasma' of monopoles and anti-monopoles screens the photon and the 'dual photon' acquires a tiny mass, $O\left(\exp -\left(M_{W} / g^{2}\right)\right)$. The 'plasma' of monopoles and anti-monopoles forms a dipole sheet across a Wilson loop to give area law.

\section{Yang-Mills theory and monopoles}

Yang-Mills theory in three Euclidean dimensions is defined as,

$$
Z=\int D \mathbf{A} \exp \left(-\frac{1}{2 g^{2}} \int d^{3} x \mathbf{B}^{a}(x) \cdot \mathbf{B}^{a}(x),\right)
$$

$\mathbf{B}^{a}(x)=\nabla \times \mathbf{A}^{a}(x)-\frac{1}{2} \varepsilon^{a b c} \mathbf{A}^{b}(x) \times \mathbf{A}^{c}(x)$, the non-Abelian magnetic field in the vector representation of the group $\mathrm{SO}(3)$. Theory is super renormalizable under the perturbation in the coupling constant $g$. Coupling constant square has a dimension of mass so naturally the perturbation theory has severe infrared divergences. However, it is expected that the theory is confining, so that there is a mass gap and no infrared divergences. This is supported by lattice gauge theory in a strong coupling expansion and also by simulations.

Yang-Mills potential in 't Hooft-Polyakov ansatz for the monopole

$$
A_{i}^{a}(x)=\varepsilon_{i a b} x^{b} \frac{1-K(r)}{r^{2}}, r=\sqrt{x^{a} x^{a}}
$$

$K(r)=1+O\left(r^{2}\right)$ as $r \rightarrow 0$ and $K(r) \rightarrow 0$ as $r \rightarrow \infty$ : this configuration is also of non zero finite action in pure Yang-Mills theory. However, there is no non-trivial classically stable configurations with a finite action. The configuration (2.2) is unstable against an indefinite expansion, as may be checked by a rescaling. We need techniques very different from the case of GGM. Nevertheless we may expect (Refs. [2], [4]-[13]) that topological configurations are relevant for the infrared behaviour of the theory. The first question is whether the configurations (2.2) have any topological significance. They have the telltale effects of a monopole on a large Wilson loop. They contribute a 
phase proportional to the solid angle subtended by the loop at the monopole (center). But we need to characterize how they are distinct from perturbative fluctuations. As a consequence, monopoles of very large size have smaller action and therefore proliferate. The situation is exactly opposite of one where the dilute gas approximation can be justified. Techniques required to handle large, light, overlapping monopole configurations and must be very different from the case of the GGM.

So to understand monopoles we should focus on the topological centers of monopoles. For a configuration with many monopoles and anti-monopoles in GGM the topological features can be located by the zeroes of the Higgs field and its behaviour in the neighborhood [14, 15]. In Ref.[16, 17] it has been proposed that for the Yang-Mills field of (2.2), the topological aspects are characterized by the degeneracies in eigenvalues of the gauge invariant symmetric matrix field, $S_{i j}(x)=\sum_{a} B_{i}^{a}(x) B_{j}^{a}(x)$,

$$
S_{i j}(x) \xi^{j A}(x)=\lambda^{A}(x) \xi^{i A}(x), \quad A=1,2,3
$$

The (anti-)monopoles can be located at points where the three eigenvalues become degenerate. At such points one of the eigenfunctions, say $A=3$, has a 'radial' behaviour.

We compare and contrast this proposal with the Abelian projection proposal of t'Hooft [15]. There a composite scalar transforming in the adjoint representation of the gauge group is formed out of the gauge field and used in place of the fundamental Higgs of the GGM. The location of the zeroes depends on the composite chosen, though the net 'monopole charge' is an invariant. In contrast we are using a gauge invariant composite (2.3) for locating the topological aspects. In spite of this difference there is a direct connection with the abelian projection proposal. We also have a closely related entity, $s^{a b}(x)=\mathbf{B}^{a}(x) \cdot \mathbf{B}^{b}(x)$,

$$
\mathbf{B}^{a}(x) \cdot \mathbf{B}^{b}(x) \xi_{b}^{A}=\lambda^{A}(x) \xi_{a}^{A}(x)
$$

which transforms homogeneously in the symmetric tensor representation of gauge group $S O(3)$. Its eigenvalues are the same as of 2.3 and hence gauge invariant. Each of the three eigenfunctions are in the adjoint representation. One of these, say $A=3$, plays the role of the scalar composite of t'Hooft. We have located the monopoles at points where the eigenvalues in (2.3) are triply degenerate. Our choice for locating the topological aspects has an added advantage. As $s^{a b}(x)$ is a symmetric (real) matrix, the three eigenfunctions $\xi_{a}^{A}(x)$ in (2.4) (after normalization) form an orthonormal set and give an $\mathrm{SO}(3)$ matrix which can be used for a local gauge transformation. Note that a given Yang-Mills field uniquely prescribes this gauge transformation (up to a trivial ambiguity in the choice of the eigenvectors). At points of degeneracy of the eigenvalues the gauge transformation is singular. In case of the 't Hooft-Polyakov ansatz (2.2), the $S O(3)$ matrix takes the form $(\hat{\theta}, \hat{\phi}, \hat{\mathbf{r}})$ i.e. the unit vectors of the spherical coordinate system $[3,14,17]$. Even though the Yang-Mills configuration (2.2) looks innocuous the transformed gauge field has singularities. Indeed, the third component (in group space) is precisely the Dirac vector potential of a monopole. The Dirac string along the third direction is due to an arbitrary choice of the 'curling' eigenvectors $\hat{\theta}, \hat{\phi}$ among the degenerate eigenvectors. Thus our gauge transformation which is dictated by the gauge configuration itself, highlights the topological aspects of the given gauge field. Even though our gauge transformation has singularities, it is an $S O(3)$ matrix at each $x$. Therefore, even though the transformed gauge potential has singularities, the transformed non-abelian field strength, which 
was finite to begin with, remains finite everywhere. This is the reason why the Dirac string does not contribute a singular term to the action. This is shown explicitly in Ref. [20].

\section{Local field theory of interactions of monopole charges and electric currents in three Euclidean dimensions}

Consider the free Maxwell theory in three Euclidean dimensions. The partition function is $Z=$ $\int D \vec{A} e^{\left(-\frac{1}{2 g^{2}} \int d^{3} x(\nabla \times \vec{A}(x))^{2}\right)}$. Using auxiliary field $\mathbf{b}$ we write $Z=\int D \mathbf{b} D \vec{A} e^{\int d^{3} x\left(-\frac{g^{2}}{2} \mathbf{b}(x)^{2}+i \mathbf{b}(x) \cdot \nabla \times \vec{A}(x)\right)}$. Note the presence of $i=\sqrt{-1}$ in the term linear in $\mathbf{a}$ in the exponent. Now the integration over $\mathbf{a}$ gives a constraint: $Z=\int D \mathbf{b} \prod_{x} \delta(\nabla \times \mathbf{b}(x)) e^{\left(-\frac{g^{2}}{2} \int d^{3} x \mathbf{b}(x)^{2}\right)}$. The constraint has the solution $\mathbf{b}(x)=$ $\nabla \chi(x)$. Therefore $Z=\int D \chi e^{\left(-\frac{g^{2}}{2} \int d^{3} x(\nabla \chi(x))^{2}\right)}$ describes a free massless scalar. This field has the interpretation as the 'dual' photon. In three dimensions the 'photon' has only one transverse degree of freedom which is described by the scalar $\chi$. In the presence of monopole sourse partition function can be written as, $\mathscr{Z}[\rho]=\int D \chi(x) e^{\int d^{3} x\left[-\frac{g^{2}}{2}(\vec{\nabla} \chi(x))^{2}+i \rho(x) \chi(x)\right]} . \rho(x)$ is the monopole density coupling locally to $\chi(x)$. Integrating over $\chi$ we can write $\mathscr{Z}[\rho]=\int D \vec{A} e^{\int d^{3} x\left[-\frac{1}{2 g^{2}}\left(\vec{\nabla} \times \vec{A}(x)-\hat{n}_{3} \partial_{3}^{-1} \rho(x)\right)^{2}\right]}$. As a consequence of this singular dislocation lines, the configurations $\vec{A}$ which matter in the functional integration, are not the usual plane waves. For the action to be finite, $\vec{\nabla} \times \vec{A}(x)$ should also be singular and cancel the Dirac string.

Consider now the interaction between sources $j$ of Dirac monopoles and electric currents $\mathbf{J}$. This is described by the partition function $Z=\int D \vec{A} D \chi e^{S_{E}+\int d^{3} x\left(i g J_{i} A_{i}+i g^{-1} j \chi\right)}$, where $S_{E}=\int d^{3} x\left(-\frac{1}{2}\left(\partial_{3} \chi\right)^{2}-\frac{1}{2}\left(\hat{\mathbf{n}}_{3} \times(\nabla \times \vec{A})\right)^{2}+i \partial_{3} \chi \hat{\mathbf{n}}_{3} \cdot \nabla \times \vec{A}\right)$. The above partition function describes the local quantum field theory involving monopole charges, photons and electric currents[19]. This is the two-potential formalism of Zwanziger for quantum electrodynamics of monopoles and charges in three Euclidean dimensions.

\section{Monopole plasma}

In Abelian theory we can construct monopole plasma by writing down Grand canonical partition function with the addition of an external chemical potential $\mu$. So the single monopole partition function will look like, $\mathscr{Z}_{1}=\int d^{3} x_{m} \xi^{3} \int \mathscr{D} \chi \mathscr{D} \vec{A} \exp \left(S_{E}+i \chi\left(x_{m}\right)-\mu\right)$ The N monopole partition function will be, $\mathscr{Z}_{N}=\int[\cdots] \int \prod_{a=1}^{N} d^{3} \vec{x}_{a} \frac{\xi^{3 N}}{N !} e^{\left[S_{E}(\chi, \vec{A})+\sum_{a} i q_{m} \chi\left(\vec{x}_{a}\right)-N \mu\right]}$, where $\xi$ is a constant and related to monopole mass. Partition function for monopole plasma can be written as

$$
\mathscr{Z}_{P}=\int \mathscr{D} \chi \mathscr{D} \vec{A} \sum_{n_{+} n_{-}} \int \prod_{a=1}^{n_{+}} d^{3} \vec{x}_{a} \prod_{b=1}^{n_{-}} d^{3} \vec{y}_{b} \frac{\zeta^{2\left(n_{+}+n_{-}\right)}}{n_{+} ! n_{-} !} e^{\left[S_{E}+\sum_{a, b} i\left(\chi\left(\vec{x}_{a}\right)-\chi\left(\vec{y}_{b}\right)\right)\right]}
$$

here $\zeta^{2}=\xi^{3} e^{-\mu}$. In Polyakov's calculation $\zeta$ is determined to be $\zeta_{P}^{2}=\frac{M_{W}^{\frac{7}{2}}}{g} \alpha\left(\frac{M_{H}}{M_{W}}\right) e^{-\frac{2 \pi M_{W}}{g^{2}} \varepsilon\left(\frac{M_{H}}{M_{W}}\right)}$ Plasma partition function can be written as $\mathscr{Z}=\int \mathscr{D} \chi \mathscr{D} \vec{A} e^{S}$

$$
S=\int d^{3} x\left(-\frac{g^{2}}{2}\left(\partial_{3} \chi\right)^{2}-\frac{1}{2 g^{2}}\left(\hat{n}_{3} \times(\nabla \times \vec{A})\right)^{2}+i \partial_{3} \chi \hat{n}_{3} \cdot \nabla \times \vec{A}+i J_{i} A_{i}+g^{2} M^{2} \cos \chi\right)
$$


where $M^{2}=\frac{2 \zeta^{2}}{g^{2}}$ The correlation functions can be calculated by expanding the $\cos \chi$ term.

$$
\begin{aligned}
g^{2}\langle\chi(-k) \chi(k)\rangle & =\frac{1}{k^{2}+M^{2}},\left\langle A_{i}(-k) \chi(k)\right\rangle=i \frac{k_{\perp i}}{k_{3}} \frac{1}{k^{2}+M^{2}}, \\
\left\langle A_{i}(-k) A_{j}(k)\right\rangle & =\frac{g^{2}}{k^{2}} \delta_{i j}+\frac{k_{\perp i} k_{\perp j}}{k_{3}^{2}}\left(\frac{g^{2}}{k^{2}}-\frac{g^{2}}{k^{2}+M^{2}}\right) \\
-\left\langle B_{i}(-k) B_{j}(k)\right\rangle & =g^{2}\left(\delta_{i j}-\frac{k_{i} k_{j}}{k^{2}+M^{2}}\right),-\left\langle B_{3}(-k) B_{3}(k)\right\rangle=\frac{g^{2} k_{\perp}^{2}}{k_{3}^{2}}\left(\frac{k_{3}^{2}+M^{2}}{k^{2}+M^{2}}\right)
\end{aligned}
$$

So all correlation functions are short ranged. However, $\left\langle B_{3}(-k) B_{3}(k)\right\rangle$ has a remnant of the Dirac string but still short ranged.

\section{Non Abelian theory}

Situation is more complicated in Yang-Mills case. We also have to handle the interactions of the monopoles with the massless charged vector bosons. The partition function can be written as, $Z=\int D \mathbf{A} e^{\left(-\frac{1}{2 g^{2}} \int d^{3} x \mathbf{B}^{a}(x) \cdot \mathbf{B}^{a}(x)\right)}, \mathbf{B}^{a}(x)=\nabla \times \mathbf{A}^{a}(x)-\frac{1}{2} \varepsilon^{a b c} \mathbf{A}^{b}(x) \times \mathbf{A}^{c}(x)$ and this can be written as,

$$
Z=\int D A_{i}^{a} D \chi^{a} e^{\int\left[-\frac{1}{2 g^{2}}\left(\vec{B}_{1}^{2}+\vec{B}_{2}^{2}\right)-\frac{g^{2}}{2}\left(D_{3}[A] \chi\right)^{2}+i\left(D_{3}[A] \chi\right)^{a} B_{3}^{a}\right]}
$$

This can be easily checked in $A_{3}=0$ gauge. Then $D_{3}[A] \chi \rightarrow \partial_{3} \chi$ and integrating over $\chi$ results with an innocent $\left(\operatorname{det} \partial_{3}^{2}\right)^{-\frac{1}{2}}$. This is fine even if we do not choose $A_{3}=0$ gauge. For example the local gauge transformation $V(x)=P \exp \left[-i \int_{-\infty}^{x_{3}} d x_{3}^{\prime} A_{3}\right]$ can be considered.

Under singular gauge transformation we will get Dirac string in the action. As a result the end point of the Dirac string will couple locally to $\chi$. Sum over all monopole and anti monopole contribution will produce $\cos \chi$ term in the action like Abelian theory.

\section{Measure and Massgap}

For the Semi-classical quantization about an instanton the measure can be obtained by the collective coordinate method. The quadratic terms in fluctuations about an instanton have zero modes related to translation and other continuous symmetries of the theory that are broken by the choice of position and other degrees of freedom of the instanton. The fluctuations which translate the instanton (for example) are replaced by an integration over the position of the instanton using the Faddeev-Popov trick. In pure YM theory there is no zero modes due to monopoles. However to get the integration over monopoles one can write the measure for single monopole case as,

$$
\mathscr{D} \mathbf{A}=D \mathbf{A}\left(x, x_{m}\right) \int d^{3} x_{m} \sqrt{g(\mathbf{A})}
$$

where $g_{p q}=\int d^{3} x \partial_{p} \mathbf{A}\left(x, x_{m}\right) \cdot \partial_{q} \mathbf{A}\left(x, x_{m},\right)$ and $D \mathbf{A}\left(x, x_{m}\right)$ is the measure for the sum over all configuration with monopole position fixed at $x_{m}$. This determinant will come as a coefficient in front of the $\cos \chi$ term and we can replace it by its expectation value. This will tell us the stories about mass gap. 


\section{Conclusion}

To handle confinement we need techniques to sum over topologically non-trivial configurations beyond a semi-classical approximation. Here we described topology locally and used this to get a singular gauge transformation which highlights the topological objects. We have incorporated the effects of the topological configurations in auxiliary fields of the first order formalism. This way the renormalized perturbation theory is still possible. Our description using singular configurations highlights how the topological aspects give contributions different from perturbation theory and it also helps in obtaining the functional measure. We can sum over positions of the topological objects in favour of a local action and the effects are contained in new terms involving the auxiliary fields of the first order formalism.

\section{References}

[1] A. M. Polyakov, Nucl. Phys. B120 (1977) 429.

[2] I. Kogan and A. Kovner, arXiv:hep-th/0205026.

[3] G. 't Hooft, Nucl. Phys. B79 (1974) 276; A. M. Polyakov, JETP Lett. 20 (1974) 194.

[4] K. Bardakci and S. Samuel, Phys. Rev. D18, 2849 (1979).

[5] A. Di Giacomo and V. I. Zakharov, arXiv:0806.2938 [hep-th].

[6] D. Diakanov and V. Petrov, arXiv:0809.2063 [hep-th].

[7] T. Suzuki, K. Ishiguro, Y. Koma and T. Sekido, Phys. Rev. D77, 034502 (2008) [arXiv:0706.4366 [hep-lat]].

[8] N. Chernodub and M. I. Polikarpov, arXiv:hep-th/9710205.

[9] H. Reinhardt, "Field Strength Approach to Yang-Mills Theory in Maximal Abelian Gauge ", prepared for Confinement 95, Osaka, 1995: proceedings (Eds. H. Toki et al) p. 151.

[10] K.-I. Kondo, Phys. Rev. D57, 7467 (1998) [arXiv:hep-th/9709109]; arXiv:hep-th/0702119.

[11] M. Schaden, arXiv:hep-th/0003030.

[12] D. Antonov, Europhys. Lett. 52, 54 (2000) [arXiv:hep-th/0003043]

[13] For a review, see J. Greensite, Prog. Part. Nucl. Phys. 51 (2003) 1 [arXiv:hep-lat/0301023].

[14] J. Arafune, P.G.O. Freund and C.J. Goebel, J. Math. Phys. 16, 433 (1975).

[15] G. ’t Hooft, Nucl. Phys.B190 (1981) 455.

[16] R. Anishetty, P. Majumdar and H. S. Sharatchandra, Phys. Lett. B478 (2000) 373 [arXiv:hep-th/9806148].

[17] E. Harikumar, I. Mitra and H. S. Sharatchandra, Phys. Lett. B557 (2003) 297 [arXiv:hep-th/0212234].

[18] E. Harikumar, I. Mitra and H. S. Sharatchandra, Phys. Lett. B557 (2003) 303 [arXiv:hep-th/0301045].

[19] C. Chatterjee, I. Mitra and H. S. Sharatchandra, Phys. Lett. B 710, 223 (2012) [arXiv:1111.6064 [hep-th]].

[20] E. Harikumar, I. Mitra and H. S. Sharatchandra, arXiv:hep-th/0304140.

[21] D. Zwanziger, Phys. Rev. D3 (1971) 880; R. A. Brandt, F. Neri and D. Zwanziger, Phys. Rev. Lett. 40 (1978) 147; R. A. Brandt, F. Neri and D. Zwanziger, Phys. Rev. D 19 (1979) 1153. 